\title{
In-hospital cardiac arrest in critically ill patients with covid-19: multicenter cohort study
}

\author{
Salim S Hayek, ${ }^{1}$ Samantha K Brenner, ${ }^{2,3}$ Tariq U Azam, ${ }^{1}$ Husam R Shadid, ${ }^{4}$ Elizabeth Anderson, ${ }^{1}$ \\ Hanna Berlin, ${ }^{4}$ Michael Pan, ${ }^{4}$ Chelsea Meloche, ${ }^{4}$ Rafey Feroz, ${ }^{4}$ Patrick O'Hayer, ${ }^{4}$ \\ Rayan Kaakati, ${ }^{4}$ Abbas Bitar, ${ }^{1}$ Kishan Padalia, ${ }^{4}$ Daniel Perry, ${ }^{1}$ Pennelope Blakely, ${ }^{1}$ \\ Shruti Gupta, ${ }^{5}$ Shahzad Shaefi, ${ }^{6}$ Anand Srivastava, ${ }^{7}$ David M Charytan, ${ }^{8}$ Anip Bansal, ${ }^{9}$ \\ Mary Mallappallil, ${ }^{10}$ Michal L Melamed, ${ }^{11}$ Alexandre M Shehata, ${ }^{12}$ Jag Sunderram, ${ }^{13}$ \\ Kusum S Mathews, ${ }^{14}$ Anne K Sutherland, ${ }^{15}$ Brahmajee K Nallamothu, ${ }^{1}$ David E Leaf, ${ }^{5}$ on behalf \\ of the STOP-COVID Investigators
}

For numbered affiliations see end of the article.

Correspondence to: S S Hayek shayek@med.umich.edu (or@salimhayek on Twitter: ORCID 0000-0003-0180-349X) Additional material is published online only. To view please visit the journal online.

Cite this as: $B M J$ 2020;371:m3513 http://dx.doi.org/10.1136/bmj.m3513 Accepted: 9 September 2020

\section{ABSTRACT}

\section{OBJECTIVES}

To estimate the incidence, risk factors, and outcomes associated with in-hospital cardiac arrest and cardiopulmonary resuscitation in critically ill adults with coronavirus disease 2019 (covid-19).

DESIGN

Multicenter cohort study.

SETTING

Intensive care units at 68 geographically diverse hospitals across the United States.

\section{PARTICIPANTS}

Critically ill adults (age $\geq 18$ years) with laboratory confirmed covid-19.

\section{MAIN OUTCOME MEASURES}

In-hospital cardiac arrest within 14 days of admission to an intensive care unit and in-hospital mortality.

RESULTS

Among 5019 critically ill patients with covid-19, $14.0 \%(701 / 5019)$ had in-hospital cardiac arrest, $57.1 \%(400 / 701)$ of whom received cardiopulmonary resuscitation. Patients who had in-hospital cardiac arrest were older (mean age 63 (standard deviation 14) $v 60$ (15) years), had more comorbidities, and were more likely to be admitted to a hospital with a smaller number of intensive care unit beds compared with those who did not have in-hospital cardiac arrest. Patients who received cardiopulmonary resuscitation were younger than those who did not (mean age 61 (standard deviation 14) v67 (14) years). The most

\section{WHAT IS ALREADY KNOWN ON THIS TOPIC}

Anecdotal reports of poor outcomes in critically ill patients with covid-19 who have had in-hospital cardiac arrest have prompted discussions on the futility of cardiopulmonary resuscitation in this patient population

Data are urgently needed on the incidence and outcomes of cardiac arrest in patients with covid-19

\section{WHAT THIS STUDY ADDS}

This multicenter cohort study was conducted in more than 5000 critically ill patients with covid-19 from 68 hospitals across the United States

The study found that in-hospital cardiac arrest was common in critically ill patients with covid-19

In-hospital cardiac arrest was associated with poor survival, even with cardiopulmonary resuscitation, particularly among older patients

common rhythms at the time of cardiopulmonary resuscitation were pulseless electrical activity (49.8\%, $199 / 400)$ and asystole $(23.8 \%, 95 / 400) .48$ of the 400 patients $(12.0 \%)$ who received cardiopulmonary resuscitation survived to hospital discharge, and only $7.0 \%(28 / 400)$ survived to hospital discharge with normal or mildly impaired neurological status. Survival to hospital discharge differed by age, with $21.2 \%$ $(11 / 52)$ of patients younger than 45 years surviving compared with $2.9 \%(1 / 34)$ of those aged 80 or older.

\section{CONCLUSIONS}

Cardiac arrest is common in critically ill patients with covid-19 and is associated with poor survival, particularly among older patients.

\section{Introduction}

Anecdotal reports of poor outcomes in critically ill patients with covid-19 who have had in-hospital cardiac arrest have prompted discussions on the futility of cardiopulmonary resuscitation in this patient population. ${ }^{1}$ However, data to guide these discussions are lacking. Only two limited reports of in-hospital cardiac arrest in patients with covid-19 have been published to date. A single center study from Wuhan, China included 151 patients with in-hospital cardiac arrest and found that only $3 \%$ of patients who received cardiopulmonary resuscitation survived to 30 days. $^{2} \mathrm{~A}$ single center study from New York City that included 31 patients with in-hospital cardiac arrest found that no patient survived to hospital discharge. ${ }^{3}$

As a result of the lack of comprehensive data on outcomes after in-hospital cardiac arrest in patients with covid-19, implementation of DNACPR (do not attempt cardiopulmonary resuscitation) policies in this setting has been controversial. Some have argued that DNACPR should be considered when the supply of intensive care unit beds is limited and also owing to the risk of covid-19 transmission to healthcare workers during cardiopulmonary resuscitation. ${ }^{45}$ These arguments are being made under the assumption of poor survival in patients with covid-19 after in-hospital cardiac arrest, however data to support this assumption are lacking. Such data are urgently needed to inform complex decision making by patients, family members, providers, and hospital leaders about policies for cardiopulmonary resuscitation and goals of treatment in the context of the ongoing covid-19 pandemic. 
We examined the incidence of in-hospital cardiac arrest and its outcomes in critically ill patients with covid-19 in a large, multicenter cohort study of more than 5000 patients admitted to intensive care units across the United States.

\section{Methods}

\section{Study design and oversight}

We used data from the Study of the Treatment and Outcomes in Critically Ill Patients with Covid-19 (STOP-COVID). This multicenter cohort study enrolled consecutive adults (age $\geq 18$ years) with laboratory confirmed covid-19 admitted to participating intensive care units at 68 geographically diverse hospitals across the US. ${ }^{6}$ We included patients admitted to the intensive care unit between 4 March and 1 June 2020. We followed patients until hospital discharge, death, or 1 July 2020 (when the study database for the current analysis was locked), whichever occurred first. Table S1 in the supplementary appendix shows a complete list of participating sites.

The study was approved with a waiver of informed consent by the institutional review board at each participating site and registered on ClinicalTrials.gov (NCT04343898). The authors designed the study, collected the data, and performed the analyses.

\section{Data collection}

Study staff at each site collected data by detailed chart review using a standardized case report form (supplementary material). Data were entered in a secure, web based platform (Research Electronic Data

\begin{tabular}{|l|l|l|l|l} 
Cardiac arrest with covid-19 \\
In-hospital incidence in critically ill patients
\end{tabular}

Capture-REDCap). ${ }^{7} 8$ Patient level data included baseline information on demographics, coexisting conditions, symptoms, and drugs before hospital admission; vital signs on intensive care unit admission; pharmacological and non-pharmacological treatments administered; and organ injury and support, such as the receipt of invasive mechanical ventilation and renal replacement therapy, in addition to daily laboratory and physiological data for the first 14 days after intensive care unit admission. Vital status was collected up to the time of hospital discharge. All data were validated through a series of automated verifications using REDCap's data quality module.

\section{Definitions and outcomes}

We defined in-hospital cardiac arrest as unexpected hemodynamic instability associated with the absence of a palpable pulse, for which cardiopulmonary resuscitation would normally be administered (whether or not cardiopulmonary resuscitation was actually administered). Patients whose death was expected because of progressive clinical deterioration, including those whose goals of treatment were comfort measures only, were not labeled as in-hospital cardiac arrest. Cardiopulmonary resuscitation was defined as the initiation of chest compressions alone or in conjunction with treatments such as defibrillation, epinephrine, vasopressin, amiodarone, lidocaine, or atropine. Table S2 in the supplementary appendix lists other treatments. We used a modified sequential organ failure assessment (mSOFA) score as a measure of acute illness severity on admission to the intensive care unit (table S3 in supplementary appendix). ${ }^{9-12}$ We recorded the date of in-hospital cardiac arrest, whether cardiopulmonary resuscitation was administered, its duration, other treatments used, and the initial cardiac rhythm at the time of arrest among patients who received cardiopulmonary resuscitation. We also recorded the functional outcome of cardiac arrest survivors at the time of hospital discharge using the cerebral performance category scale, which is widely used to evaluate functional outcomes in resuscitation research. ${ }^{1314}$

\section{Statistical analysis}

We report the clinical characteristics of patients stratified by in-hospital cardiac arrest, whether they received cardiopulmonary resuscitation, and whether they survived to hospital discharge. Continuous variables are presented as mean (standard deviation) or as median (25th-75th interquartile range) for normally and non-normally distributed data, respectively. Categorical variables are presented as count and percentage. We used the test to compare normally distributed variables and the MannWhitney U or Kruskal-Wallis tests to compare nonnormally distributed variables between groups. We used multivariable logistic regression to identify independent predictors of in-hospital cardiac arrest and to identify independent predictors of not receiving cardiopulmonary resuscitation. The following 
covariates were selected for inclusion in the model based on clinical knowledge and biological plausibility: age, sex, race, history of smoking, body mass index, diabetes mellitus, hypertension, coronary artery disease, congestive heart failure, chronic obstructive pulmonary disease, active malignancy, chronic or end stage kidney disease, mSOFA score, and the number of intensive care unit beds at the admitting hospitals before covid-19 (not including surge capacity beds). Table S4 in the supplementary appendix reports counts and frequencies for missing values. Missing data were not imputed; multivariable models used complete case analysis. All analyses were performed by using SPSS 24 (IBM, NY, USA).

\section{Patient and public involvement}

The multicenter study design and the need to generate data quickly in the midst of the pandemic precluded patient or public involvement in the design or conduct of the study.

\section{Results}

Baseline characteristics, predictors, and outcomes of cardiac arrest

Among 5019 patients included in the study, 701 $(14.0 \%)$ had in-hospital cardiac arrest within 14 days of admission to the intensive care unit (fig 1, table 1). The median time from hospital admission to in-hospital cardiac arrest was seven days (interquartile range 4-11 days); $15.3 \%$ (107/701) of patients had in-hospital cardiac arrest on the day of admission to the intensive care unit. Patients who had in-hospital cardiac arrest were more likely to be older, to be admitted to a hospital with a smaller number of intensive care unit beds, and to have a higher burden of cardiovascular disease and risk factors (tobacco use, diabetes mellitus, hypertension, coronary artery disease, chronic or end stage kidney disease) compared with patients who did not have inhospital cardiac arrest (table 1). These patients also had more pronounced laboratory abnormalities on the day of admission to the intensive care unit and were more likely to have received two or more vasopressors compared with those who did not have in-hospital cardiac arrest (table 1).

In multivariable analysis, several characteristics were associated with a higher risk of in-hospital cardiac arrest: being admitted to a hospital with a smaller number of intensive care unit beds, older age, black race, and higher mSOFA score on admission to the intensive care unit (fig 2). Coronary artery disease and congestive heart failure were not associated with in-hospital cardiac arrest (fig 2). Overall, 653 of 701 patients (93.2\%) with in-hospital cardiac arrest died, including 301 who died without receiving cardiopulmonary resuscitation and 352 who died despite cardiopulmonary resuscitation (fig 1).

\section{Characteristics and outcomes of cardiopulmonary resuscitation}

Among the 701 patients with in-hospital cardiac arrest, $400(57.1 \%)$ received cardiopulmonary resuscitation (fig 1). The 42.9\% (301/701) of patients who did not receive cardiopulmonary resuscitation had a DNACPR code status at the time of in-hospital cardiac arrest. Patients who received cardiopulmonary resuscitation were younger than those who did not (mean age 61 (standard deviation 14) v 67 (14) years) and were less likely to be receiving invasive mechanical

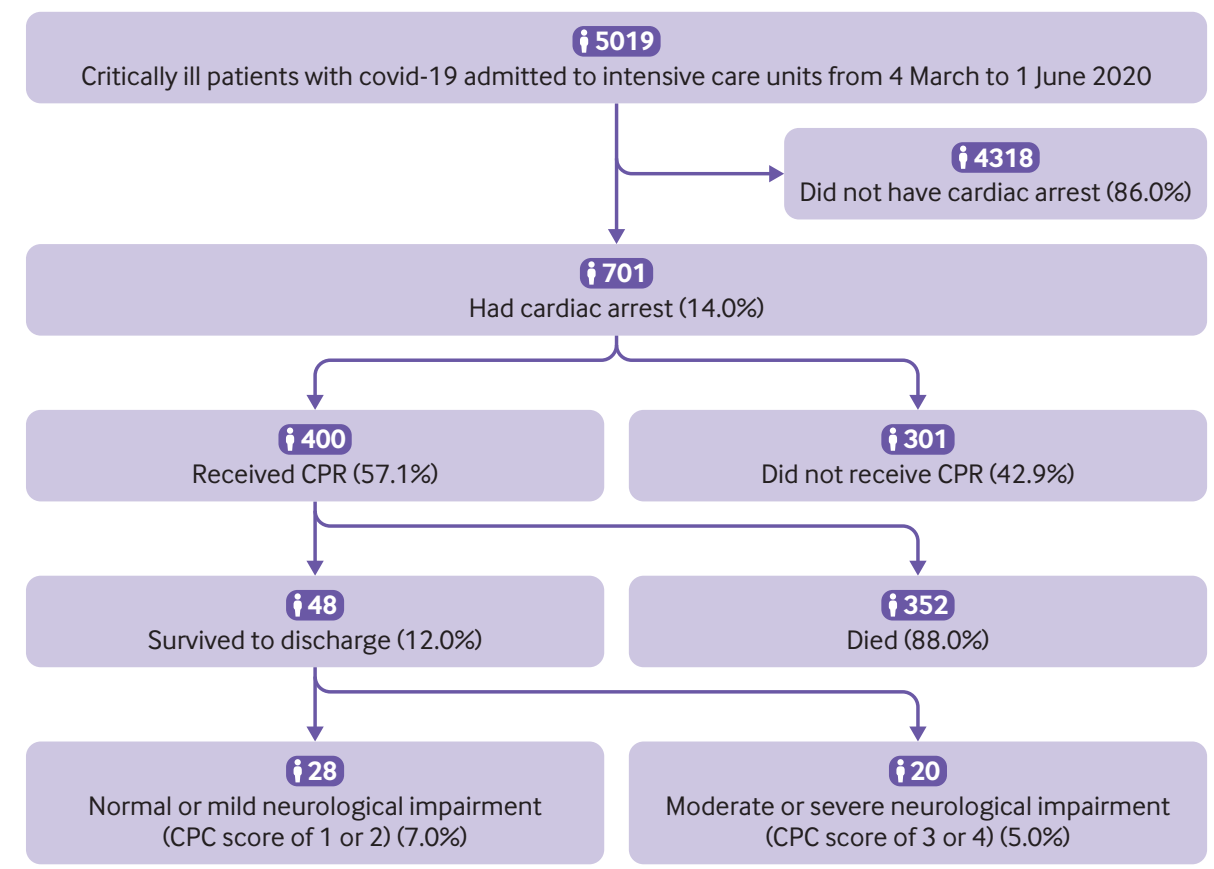

Fig 1 | Flow diagram depicting study population, incidence of in-hospital cardiac arrest, cardiopulmonary resuscitation, and outcomes. Percentages in lower boxes relate to group who underwent CPR $(n=400)$. Covid$19=$ coronavirus disease 2019; $C P C=$ cerebral performance category; $C P R=$ cardiopulmonary resuscitation 


\begin{tabular}{|c|c|c|c|}
\hline Characteristic & No cardiac arrest $(n=4318,86.0 \%)$ & Cardiac arrest $(n=701,14.0 \%)$ & $P$ value \\
\hline \multicolumn{4}{|l|}{ ICU beds } \\
\hline$<50$ & $1268 / 4318(29.4)$ & 434/701 (61.9) & $<0.001$ \\
\hline $50-99$ & $1248 / 4318(28.9)$ & $144 / 701(20.5)$ & 0.031 \\
\hline$\geq 100$ & $1802 / 4318(41.7)$ & $123 / 701(17.5)$ & $<0.001$ \\
\hline \multicolumn{4}{|l|}{ Demographics } \\
\hline Age (years; mean (SD)) & $60(15)$ & $63(14)$ & $<0.001$ \\
\hline$<45$ & $647 / 4318(15.0)$ & $68 / 701(9.7)$ & $<0.001$ \\
\hline $45-64$ & $1851 / 4318(42.9)$ & $277 / 701(39.5)$ & 0.003 \\
\hline $65-79$ & $1419 / 4318(32.9)$ & $270 / 701(38.5)$ & $<0.001$ \\
\hline$\geq 80$ & $401 / 4318(9.3)$ & $86 / 701(12.3)$ & $<0.001$ \\
\hline Male sex & $2707 / 4318(62.7)$ & $458 / 701(65.3)$ & 0.15 \\
\hline \multicolumn{4}{|l|}{ Race } \\
\hline White (non-Hispanic) & $1263 / 4318(29.2)$ & $150 / 701(21.4)$ & $<0.001$ \\
\hline Black (non-Hispanic) & $1280 / 4318(29.6)$ & $226 / 701(32.2)$ & 0.17 \\
\hline Hispanic & $484 / 4318(11.2)$ & $67 / 701(9.6)$ & 0.22 \\
\hline Asian & $248 / 4318(5.7)$ & 43/701 (6.1) & 0.66 \\
\hline Unknown & $1043 / 4318(24.2)$ & $215 / 701(30.7)$ & $<0.001$ \\
\hline Body mass index (mean (SD))* & $32(8)$ & $32(9)$ & 0.23 \\
\hline$<30$ & $1725 / 4165(41.4)$ & $283 / 656(43.1)$ & 0.044 \\
\hline $30-34$ & $1111 / 4165(26.7)$ & $189 / 656(28.8)$ & 0.72 \\
\hline $35-39$ & $640 / 4165(15.4)$ & $76 / 656(11.6)$ & 0.007 \\
\hline$\geq 40$ & $689 / 4165(16.5)$ & $108 / 656(16.5)$ & 0.59 \\
\hline \multicolumn{4}{|l|}{ Coexisting conditions and risk factors $t$} \\
\hline Current or former tobacco use ${ }^{\star}$ & $1861 / 3756(49.5)$ & $313 / 564(55.5)$ & 0.007 \\
\hline Diabetes mellitus & $1770 / 4318(41.0)$ & $340 / 701(48.5)$ & $<0.001$ \\
\hline Hypertension & $2611 / 4318(60.5)$ & $475 / 701(67.8)$ & $<0.001$ \\
\hline Coronary artery disease & $563 / 4318(13.0)$ & $113 / 701(16.1)$ & 0.001 \\
\hline Congestive heart failure & $434 / 4318(10.1)$ & $78 / 701(11.1)$ & 0.17 \\
\hline Chronic obstructive pulmonary disease & $377 / 4318(8.7)$ & $56 / 701(8.0)$ & 0.52 \\
\hline Chronic or end stage kidney disease & $671 / 4318(15.5)$ & $148 / 701(21.1)$ & $<0.001$ \\
\hline Active malignancy & $188 / 4318(4.4)$ & $39 / 701(5.6)$ & 0.07 \\
\hline \multicolumn{4}{|c|}{ Laboratory values on day of ICU admission (median (IQR)) } \\
\hline White cell count $($ per mm³) & $8.2(5.9-11.5)$ & $10.1(7-14.3)$ & $<0.001$ \\
\hline Lymphocyte count (per mm³) & $10.1(6.2-15.5)$ & $8.3(4.8-13.6)$ & $<0.001$ \\
\hline Hemoglobin $(\mathrm{g} / \mathrm{dL})$ & $12.6(14-11)$ & $12.3(10.6-13.8)$ & 0.009 \\
\hline Platelet count $\left(10^{3} / \mu \mathrm{L}\right)$ & $214(163-276)$ & $222(165-285)$ & 0.16 \\
\hline Creatinine $(\mathrm{mg} / \mathrm{dL})$ & $1.03(0.79-1.59)$ & $1.4(0.92-2.49)$ & $<0.001$ \\
\hline Albumin $(\mathrm{g} / \mathrm{dL})$ & $3.2(2.8-3.6)$ & $3.1(2.7-3.5)$ & $<0.001$ \\
\hline Aspartate aminotransferase $(\mathrm{U} / \mathrm{L})$ & $51(35-80)$ & $64(39-109)$ & $<0.001$ \\
\hline Alanine aminotransferase (U/L) & $35(22-59)$ & $40(24-68)$ & $<0.001$ \\
\hline Total bilirubin $(\mathrm{mg} / \mathrm{dL})$ & $0.6(0.4-0.8)$ & $0.6(0.4-0.9)$ & $<0.001$ \\
\hline Lactate $(\mathrm{mmol} / \mathrm{L})$ & $1.5(1.1-2.2)$ & $2.1(1.3-3.1)$ & $<0.001$ \\
\hline Arterial pH & $7.38(7.31-7.44)$ & $7.33(7.239-7.41)$ & $<0.001$ \\
\hline D dimer $(\mathrm{ng} / \mathrm{mL})$ & $1142(610-2800)$ & $2380(1220-8200)$ & $<0.001$ \\
\hline C reactive protein $(\mathrm{mg} / \mathrm{L})$ & $146(77.5-225)$ & $173(95.3-261)$ & $<0.001$ \\
\hline Ferritin (ng/mL) & $938(464-1843)$ & $1234(604-2653)$ & $<0.001$ \\
\hline mSOFA score on ICU admission (mean (SD)) & $4.1(3.1)$ & $6.0(3.3)$ & $<0.001$ \\
\hline \multicolumn{4}{|l|}{ Complications $\neq$} \\
\hline Invasive mechanical ventilation & $3172 / 4318(73.5)$ & 491/701 (70.0) & 0.029 \\
\hline Extracorporeal membrane oxygenation & $174 / 4318(4.0)$ & $2 / 701(0.3)$ & $<0.001$ \\
\hline Newly diagnosed reduced left ventricular function§ & $118 / 4318(2.7)$ & 48/701 (6.8) & $<0.001$ \\
\hline Receipt of at least two vasopressors & $1255 / 4318(29.1)$ & $362 / 701(51.6)$ & $<0.001$ \\
\hline Acute kidney injury requiring RRT & $842 / 4318(19.5)$ & $161 / 701(23.0)$ & 0.033 \\
\hline \multicolumn{4}{|l|}{ Outcomes } \\
\hline ICU length of stay (days; median (IQR)) & $17(9-30)$ & $6(4-10)$ & $<0.001$ \\
\hline Survived and discharged from hospital & $2759 / 4318(63.9)$ & 48/701 (6.8) & $<0.001$ \\
\hline Died while in hospital & $1390 / 4318(32.2)$ & $653 / 701(93.2)$ & $<0.001$ \\
\hline Still in hospital 30 days after ICU admission & $169 / 4318(3.9)$ & $0 / 701(0.0)$ & - \\
\hline \multicolumn{4}{|c|}{$\begin{array}{l}\text { ICU=intensive care unit; IQR=interquartile range; mSOFA=modified sequential organ failure assessment score; RRT=renal replacement therapy; SD=standard deviation. } \\
\text { *Denominator differs in variables for smoking and body mass index because of missing data for these specific variables (listed in supplementary appendix). } \\
\text { tDefinitions of coexisting conditions are provided in supplementary appendix. } \\
\text { fOn ICU admission for non-cardiac arrest group, or before cardiac arrest. } \\
\text { §Defined as newly diagnosed left ventricular ejection fraction less than } 50 \% \text { on echocardiogram. }\end{array}$} \\
\hline
\end{tabular}

ventilation at the time of in-hospital cardiac arrest (table 2). In multivariable analysis, only older age was independently associated with a higher likelihood of not receiving cardiopulmonary resuscitation (fig 2). Rates of cardiopulmonary resuscitation use differed considerably by age: $76.5 \%(52 / 68)$ of patients 
younger than 45 years received cardiopulmonary resuscitation and only $39.5 \%$ (34/86) of patients aged 80 and older received cardiopulmonary resuscitation (fig 3).

The most common initial cardiac rhythms at the time of cardiopulmonary resuscitation were pulseless electrical activity $(49.8 \%, 199 / 400)$, asystole $(23.8 \%$, 95/400), ventricular tachycardia (8.3\%, 33/400), and ventricular fibrillation $(3.8 \%, 15 / 400)$. Pulseless electrical activity was more common in patients who survived to discharge compared with those who died, whereas the distribution of other rhythms was similar between survivors and non-survivors (table 3). Epinephrine was the most commonly used treatment during cardiopulmonary resuscitation $(81.0 \%$, $324 / 400)$, followed by defibrillation (18.5\%, 74/400). The median duration of cardiopulmonary resuscitation was 10 minutes (interquartile range 5-18). Younger patients received cardiopulmonary resuscitation for a longer duration than older patients (median 13 minutes (interquartile range 7-20) in patients younger than 45 years compared with 7 minutes (4-14) in patients aged 80 or older).

Overall, 135 of the 400 patients (33.8\%) who received cardiopulmonary resuscitation achieved return of spontaneous circulation, but only $12.0 \%$
(48/400) survived to hospital discharge. Return of spontaneous circulation was achieved at a median time of 6 minutes (interquartile range 4-14) after initiation of cardiopulmonary resuscitation. In those who died after return of spontaneous circulation, the median time from cardiopulmonary resuscitation to death was 7 days (interquartile range 3-15 days). The likelihood of survival to hospital discharge decreased with older age, ranging from 21.2\% (11/52) among patients younger than 45 years to $2.9 \%$ (1/34) among patients aged 80 and older (fig 3 ). Duration of cardiopulmonary resuscitation did not differ significantly according to sex, race, or number of intensive care unit beds (table S5 in supplementary appendix). In multivariable analysis, age 80 or older, male sex, and admission to a hospital with a smaller number of intensive care unit beds were each independently associated with an increased risk of death after cardiopulmonary resuscitation, whereas mSOFA score was not (table S6 in supplementary appendix). Among the 48 patients who survived to hospital discharge, 58.3\% (28/48) had normal or mildly impaired neurological status (cerebral performance category score of 1 to 2), and $41.7 \%$ (20/48) had moderate to severe neurological dysfunction (cerebral performance category score of 3 or 4; fig 1).

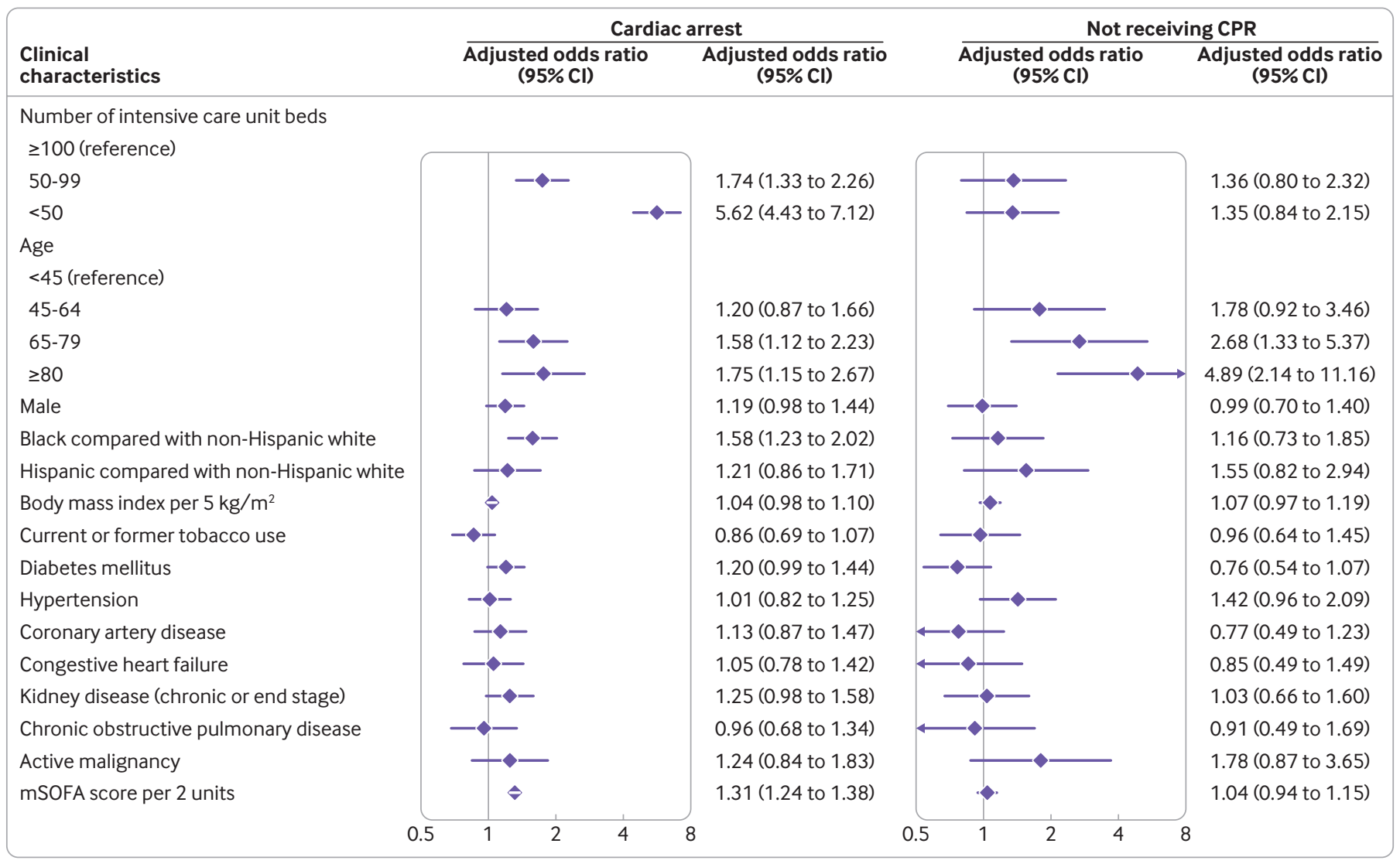

Fig 2 | Determinants of cardiac arrest and not receiving cardiopulmonary resuscitation in critically ill patients with coronavirus disease 2019. Forest plot depicting adjusted odds ratios and $95 \%$ confidence intervals for two separate binary logistic regression models: association with in-hospital cardiac arrest, and association with not receiving cardiopulmonary resuscitation. $C P R=$ cardiopulmonary resuscitation; $\mathrm{mSOFA}=\mathrm{modified}$ sequential organ failure assessment 


\begin{tabular}{|c|c|c|c|}
\hline Characteristic & Received CPR $(n=400,57.1 \%)$ & Did not receive CPR $(n=301,42.9 \%)$ & P value \\
\hline \multicolumn{4}{|l|}{ ICU beds } \\
\hline$<50$ & $237 / 400(59.3)$ & $197 / 301(65.4)$ & 0.10 \\
\hline $50-99$ & $82 / 400(20.5)$ & $62 / 301(20.6)$ & 0.99 \\
\hline$\geq 100$ & $81 / 400(20.3)$ & 42/301 (14.0) & 0.04 \\
\hline \multicolumn{4}{|l|}{ Demographics } \\
\hline Age (years; mean (SD)) & $61(14)$ & $67(14)$ & $<0.001$ \\
\hline$<45$ & $52 / 400(13.0)$ & $16 / 301(5.3)$ & 0.001 \\
\hline $45-64$ & $172 / 400(43.0)$ & $105 / 301(34.9)$ & 0.04 \\
\hline $65-79$ & $142 / 400(39.5)$ & $128 / 301(42.5)$ & 0.06 \\
\hline$\geq 80$ & $34 / 400(8.5)$ & $52 / 301(17.3)$ & 0.001 \\
\hline Male sex & $266 / 400(66.5)$ & $192 / 301(63.8)$ & 0.46 \\
\hline \multicolumn{4}{|l|}{ Race } \\
\hline White (non-Hispanic) & $84 / 400(21.0)$ & 66/301 (21.9) & 0.78 \\
\hline Black (non-Hispanic) & $131 / 400(32.8)$ & $95 / 301(31.6)$ & 0.75 \\
\hline Hispanic & $38 / 400(9.5)$ & 29/301 (9.6) & 0.99 \\
\hline Asian & $26 / 400(6.5)$ & $17 / 301(5.6)$ & 0.75 \\
\hline Unknown & $121 / 400(30.3)$ & $94 / 301(31.2)$ & 0.80 \\
\hline Body mass index (mean (SD))* & $32(8)$ & $32(10)$ & 0.92 \\
\hline$<30$ & $150 / 366(41.0)$ & $133 / 290(45.9)$ & 0.23 \\
\hline $30-34$ & $114 / 366(31.1)$ & $75 / 290(25.9)$ & 0.14 \\
\hline $35-39$ & $47 / 366(12.8)$ & $29 / 290(10.0)$ & 0.27 \\
\hline$\geq 40$ & $55 / 366(15.0)$ & $53 / 290(18.3)$ & 0.29 \\
\hline \multicolumn{4}{|l|}{ Coexisting conditions and risk factors $\dagger$} \\
\hline Current or former tobacco use ${ }^{\star}$ & $171 / 327(52.3)$ & $142 / 237(59.9)$ & 0.46 \\
\hline Diabetes mellitus & $201 / 400(50.2)$ & $139 / 301(46.2)$ & 0.29 \\
\hline Hypertension & $253 / 400(63.2)$ & $222 / 301(73.8)$ & 0.003 \\
\hline Coronary artery disease & $67 / 400(16.8)$ & $46 / 301(15.3)$ & 0.60 \\
\hline Congestive heart failure & $47 / 400(11.8)$ & $31 / 301(10.3)$ & 0.55 \\
\hline Chronic obstructive pulmonary disease & $31 / 400(7.8)$ & $25 / 301(8.3)$ & 0.79 \\
\hline Chronic or end stage kidney disease & $84 / 400(21.0)$ & $64 / 301(21.3)$ & 0.93 \\
\hline Active malignancy & $17 / 400(4.3)$ & $22 / 301(7.3)$ & 0.08 \\
\hline \multicolumn{4}{|c|}{ Laboratory values on day of ICU admission (median (IQR)) } \\
\hline White cell count (per $\mathrm{mm}^{3}$ ) & $10.1(7.0-14.3)$ & $10.1(7.1-14.4)$ & 0.70 \\
\hline Lymphocyte count (per mm³) & $9.0(5.0-14.0)$ & $7.3(4.6-12.7)$ & 0.05 \\
\hline Hemoglobin $(\mathrm{g} / \mathrm{dL})$ & $12.5(10.6-13.8)$ & $12.3(10.6-13.7)$ & 0.57 \\
\hline Platelet count $\left(10^{3} / \mu \mathrm{L}\right)$ & $217(168-284)$ & $226(159-291)$ & 0.99 \\
\hline Creatinine $(\mathrm{mg} / \mathrm{dL})$ & $1.30(0.90-2.40)$ & $1.51(0.96-2.49)$ & 0.08 \\
\hline Albumin $(\mathrm{g} / \mathrm{dL})$ & $3.1(2.7-3.5)$ & $3.1(2.7-3.5)$ & 0.94 \\
\hline Aspartate aminotransferase $(\mathrm{U} / \mathrm{L})$ & $65(41-120)$ & $62(36-99)$ & 0.15 \\
\hline Alanine aminotransferase $(\mathrm{U} / \mathrm{L})$ & $42(25-76)$ & $39(23-59)$ & 0.04 \\
\hline Total bilirubin $(\mathrm{mg} / \mathrm{dL})$ & $0.6(0.4-0.9)$ & $0.6(0.4-0.9)$ & 0.75 \\
\hline Lactate $(\mathrm{mmol} / \mathrm{L})$ & $2.2(1.3-3.4)$ & $2(1.4-2.9)$ & 0.22 \\
\hline Arterial pH & $7.32(7.22-7.44)$ & $7.35(7.25-7.42)$ & 0.07 \\
\hline D dimer $(\mathrm{ng} / \mathrm{mL})$ & $2637(1283-8680)$ & $2180(1160-7955)$ & 0.44 \\
\hline C reactive protein $(\mathrm{mg} / \mathrm{L})$ & $157(83-249)$ & $190.9(110-269)$ & 0.04 \\
\hline Ferritin $(\mathrm{ng} / \mathrm{mL})$ & $1225(636-2591)$ & $1255(599.43-2784)$ & 0.84 \\
\hline \multicolumn{4}{|l|}{ mSOFA score on ICU admission } \\
\hline Mean (SD) & $5.9(3.3)$ & $6.2(3.3)$ & 0.26 \\
\hline \multicolumn{4}{|l|}{ Complications $\neq$} \\
\hline Invasive mechanical ventilation & $257 / 400(64.3)$ & $234 / 301(77.7)$ & $<0.001$ \\
\hline On extracorporeal membrane oxygenation & $13 / 400(3.3)$ & $1 / 301(0.3)$ & $<0.001$ \\
\hline Newly diagnosed reduced left ventricular function§ & $29 / 400(7.2)$ & $19 / 301(6.3)$ & 0.70 \\
\hline Receipt of at least two vasopressors & $226 / 400(56.5)$ & $197 / 301(65.4)$ & 0.001 \\
\hline Acute kidney injury requiring RRT & $78 / 400(19.5)$ & $83 / 301(27.6)$ & 0.01 \\
\hline \multicolumn{4}{|c|}{$\begin{array}{l}\text { ICU=intensive care unit; IQR=interquartile range; mSOFA=modified sequential organ failure assessment score; RRT=renal replacement therapy; SD=standard deviation. } \\
\text { *Denominator differs in variables for smoking and body mass index because of missing data for these specific variables (listed in supplementary appendix). } \\
\text { †Definitions of coexisting conditions are provided in supplementary appendix. } \\
\text { †Before cardiac arrest. } \\
\text { §Defined as newly diagnosed left ventricular ejection fraction less than } 50 \% \text { on echocardiogram. }\end{array}$} \\
\hline
\end{tabular}

\section{Discussion}

Principal findings

In a large multicenter study of more than 5000 critically ill adults with covid-19 admitted to intensive care units at 68 participating hospitals across the US, we found that approximately one in seven patients experienced in-hospital cardiac arrest, of whom only $57.1 \%$ (400/701) received cardiopulmonary resuscitation. Among those who received cardiopulmonary resuscitation, only $12.0 \%(48 / 400)$ survived 


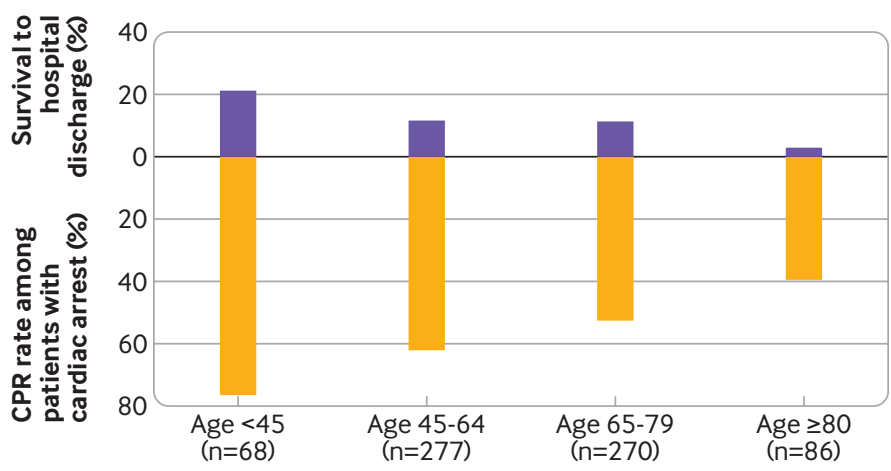

Fig 3 | Proportion of patients with cardiac arrest who underwent cardiopulmonary resuscitation, and of those, proportion who survived to hospital discharge, stratified by age. Number of patients with cardiac arrest given for each age category (eg, among patients $<45$ years old, 68 had cardiac arrest, $76.5 \%$ of whom received cardiopulmonary resuscitation; $21.2 \%$ survived to hospital discharge). CPR=cardiopulmonary resuscitation not captured in our database could have had a major impact on outcomes. Cumulatively, these data indicate poor outcomes in critically ill patients with in-hospital cardiac arrest, particularly in older patients.

\section{Implications}

Three important implications can be reported from our findings. Firstly, a substantial number of patients who are critically ill with covid-19 will suffer cardiac arrest. Although this had been anecdotally reported, we offer large scale data from multiple US hospitals on this topic. This finding is important and should prompt clinicians to design systems that are able to accommodate for large numbers of these events during future outbreaks, including methods to protect frontline healthcare providers from infection. Secondly, we identified patient and hospital specific risk factors for cardiac arrest, thereby highlighting those at greatest risk who might require closer monitoring or earlier goals of treatment discussions. Finally, our work better characterizes the likelihood of survival after in-hospital cardiac arrest specific to covid-19 related critical illness. Information on survival will provide clinicians, patients, and families with real world information on outcomes after these events. This information could guide end-of-life care discussions with critically ill patients with covid-19 and their families.

Our study has additional findings of interest. Cardiac arrest in critically ill patients with covid-19 appears of in-hospital cardiac arrest, suggesting that hospita resources, staffing, expertise, strain, or other factors

to hospital discharge, and only $7.0 \%$ (28/400) did so with normal or mildly impaired neurological status. forischarge varied considerably gears patients who survived to hospital discharge required only a short course of cardiopulmonary resuscitation. Admission to a hospital with fewer intensive care unit beds was strongly associated with a greater risk

\begin{tabular}{|c|c|c|c|}
\hline Characteristic & Died $(n=352,88.0 \%)$ & Survived to discharge $(n=48,12.0 \%)$ & $P$ value \\
\hline \multicolumn{4}{|l|}{ ICU beds } \\
\hline$<50$ & $226 / 352(64.2)$ & $11 / 48(22.9)$ & $<0.001$ \\
\hline $50-99$ & $70 / 352$ (19.9) & $12 / 48(25.0)$ & 0.45 \\
\hline$\geq 100$ & $56 / 352(15.9)$ & $25 / 48(52.1)$ & $<0.001$ \\
\hline \multicolumn{4}{|l|}{ Demographics } \\
\hline Age (years; mean (SD)) & $61(14)$ & $57(15)$ & 0.03 \\
\hline Male sex & $242 / 352(68.8)$ & $24 / 48(50.0)$ & 0.01 \\
\hline \multicolumn{4}{|l|}{ Race } \\
\hline White (non-Hispanic) & $71 / 352(20.2)$ & $13 / 48(27.1)$ & 0.26 \\
\hline Black (non-Hispanic) & $116 / 352(33.0)$ & $15 / 48(31.3)$ & 0.87 \\
\hline Hispanic & $33 / 352(9.4)$ & $5 / 48(10.4)$ & 0.79 \\
\hline Asian & $21 / 352(6.0)$ & $5 / 48(10.4)$ & 0.22 \\
\hline Unknown & $111 / 352(31.5)$ & $10 / 48(20.8)$ & 0.18 \\
\hline \multicolumn{4}{|l|}{ Initial cardiac rhythm } \\
\hline Ventricular fibrillation & $13 / 352(3.7)$ & $2 / 48(4.2)$ & 0.69 \\
\hline Pulseless ventricular tachycardia & $30 / 352(8.5)$ & $3 / 48(6.3)$ & 0.78 \\
\hline Pulseless electrical activity & $169 / 352(48.0)$ & $30 / 48(62.5)$ & 0.04 \\
\hline Asystole & $89 / 352(25.3)$ & $6 / 48(12.5)$ & 0.07 \\
\hline Unknown & $45 / 352(14.5)$ & $7 / 48(14.6)$ & 0.82 \\
\hline \multicolumn{4}{|c|}{ Treatments administered during CPR } \\
\hline Defibrillation & 68/352 (19.3) & $6 / 48(12.5)$ & 0.25 \\
\hline Epinephrine & $291 / 352(82.7)$ & $33 / 48(68.8)$ & 0.02 \\
\hline Vasopressin & $17 / 352(4.8)$ & $2 / 48(4.2)$ & 0.84 \\
\hline Amiodarone & $31 / 352(8.8)$ & $3 / 48(6.3)$ & 0.55 \\
\hline Lidocaine & $9 / 352(2.6)$ & $0 / 48(0.0)$ & 0.26 \\
\hline Atropine & $18 / 352(5.1)$ & $2 / 48(4.2)$ & 0.78 \\
\hline Chest compressions alone & 39/352 (11.1) & $8 / 48(16.7)$ & 0.26 \\
\hline \multicolumn{4}{|l|}{ Duration of CPR (min) } \\
\hline Median (IQR) & $12(6-19)$ & $4(2-6)$ & $<0.001$ \\
\hline
\end{tabular}


to be predominantly related to non-cardiac causes because the initial rhythm for most patients in our study was non-shockable (pulseless electrical activity or asystole in $73 \%$ of patients). Respiratory failure and prothrombotic events that have been extensively described in patients with covid-19 are probably major contributors to in-hospital cardiac arrest in this setting. ${ }^{15-17}$ Shao and colleagues reported similar findings in their study of 151 patients with in-hospital cardiac arrest in Wuhan, China. They found $87.5 \%$ of patients had a respiratory cause for their cardiac arrest, and asystole was the initial cardiac rhythm in $89 \%$ of patients. ${ }^{2}$ Consistent with these findings, we observed that coronary artery disease and congestive heart failure were not independently associated with cardiac arrest. This finding suggests that severity of the acute illness rather than comorbidities plays a more dominant role in determining in-hospital cardiac arrest in this population.

Another striking finding from our study is that only about half of the patients who experienced cardiac arrest received cardiopulmonary resuscitation compared with $90 \%$ of patients in the study by Shao and colleagues. ${ }^{2}$ Several possible explanations could explain this difference. Most of the patients in the study by Shao and colleagues were on general medical floors and were probably considered less likely to die than the patients in the current study, all of whom were admitted to intensive care units. Other potential reasons for the large disparity in rates of cardiopulmonary resuscitation between studies include differences in age, comorbidity burden, cultural preferences, and clinician estimations of futility. Patients in our study who did not receive cardiopulmonary resuscitation had a DNACPR order in place. We did not document the date or the reasons why DNACPR status was implemented, but none of the centers in STOP-COVID had an institutional policy in place for routinely withholding cardiopulmonary resuscitation from patients with covid-19. However, many centers adopted a proactive approach involving palliative care teams and initiation of early goals of care discussions with patients and family members, which could have led to the implementation of DNACPR orders for many of these critically ill patients.

Among those who received cardiopulmonary resuscitation in our study, $12.0 \%$ survived to hospital discharge. These outcomes are better than the 3\% survival at 30 days reported by Shao and colleagues, ${ }^{2}$ and are consistent with those recently reported in a study of critically ill patients with non-covid-19 disease from the GWTG-R registry. ${ }^{18}$ Overall, these findings suggest that patients with covid-19 with cardiac arrest had outcomes at these hospitals that were similar to critical illnesses like sepsis, pneumonia, and acute respiratory distress syndrome even during early stages of the pandemic. Baseline information from our study will be valuable for benchmarking and tracking hospital outcomes, and planning local policies around resuscitation as new treatments might shift decision making in future waves of the pandemic.

\section{Strengths and limitations}

Our study has several strengths. It is a large study of cardiac arrest and cardiopulmonary resuscitation outcomes in patients with covid-19. All data were extracted by detailed chart review, with extensive and granular data collected from 68 geographically diverse centers. All patients were followed until death, hospital discharge, or a minimum of 30 days from admission to the intensive care unit. Additionally, we collected data on neurological status at hospital discharge among survivors.

We also acknowledge several limitations. Firstly, data on the cardiac rhythm of patients who did not receive cardiopulmonary resuscitation were not available. Secondly, we were unable to capture the timing of initiation of treatments such as chest compressions or defibrillation. Consequently, we were unable to assess the quality and timeliness of resuscitation, which can be compromised in patients with covid-19 owing to the requirements for donning personal protective equipment, and could have contributed to the poor outcomes. Thirdly, the data were collected during the early months of the pandemic in the US and might not reflect current trends, including mortality rates given the application of new treatments (eg, dexamethasone). Fourthly, to minimize data entry burden on the participating centers in the midst of the pandemic, laboratory and acute organ injury data, including cardiac arrest, were limited to the first 14 days after admission to the intensive care unit, which could have led to underestimation of the true incidence of cardiac arrest.

\section{Conclusions}

Cardiac arrest is common in critically ill patients with covid-19 and is associated with poor survival even when cardiopulmonary resuscitation is provided, particularly in patients aged 80 or older, and in those requiring prolonged cardiopulmonary resuscitation. Our study data could help inform patients, family members, and clinicians in complex decision making about patients with covid-19 who are at risk of cardiac arrest or who have experienced cardiac arrest.

\section{AUTHOR AFFILIATIONS}

${ }^{1}$ Division of Cardiology, Department of Medicine, University of Michigan, Frankel Cardiovascular Center, 1500 East Medical Center Drive, Ann Arbor, Ml 48109, USA

${ }^{2}$ Department of Internal Medicine, Hackensack Meridian School of Medicine at Seton Hall, Nutley, NJ, USA

${ }^{3}$ Department of Internal Medicine, Heart and Vascular Hospital, Hackensack Meridian Health Hackensack University Medical Center, Hackensack, NJ, USA

${ }^{4}$ Department of Medicine, University of Michigan, Ann Arbor, MI, USA

${ }^{5}$ Division of Renal Medicine, Brigham and Women's Hospital, Boston, MA, USA

${ }^{6}$ Department of Anesthesia, Critical Care and Pain Medicine, Beth Israel Deaconess Medical Center, Harvard Medical School, Boston, MA, USA

${ }^{7}$ Center for Translational Metabolism and Health, Institute for Public Health and Medicine, Division of Nephrology and Hypertension, Northwestern University Feinberg School of Medicine, Chicago, IL, USA 
${ }^{8}$ Division of Nephrology, New York University Grossman School of Medicine, New York, NY, USA

${ }^{9}$ Division of Renal Diseases and Hypertension, University of Colorado, Anschutz Medical Campus, Aurora, CO, USA

${ }^{10}$ Division of Nephrology, Department of Internal Medicine, NYC Health + Hospitals Kings County Hospital Center, Brooklyn, NY, USA

${ }^{11}$ Division of Nephrology, Department of Medicine, Albert Einstein College of Medicine, Montefiore Medical Center, Bronx, NY, USA

${ }^{12}$ Hackensack Meridian Health Mountainside Medical Center, Glen Ridge, NJ, USA

${ }^{13}$ Division of Pulmonary and Critical Care Medicine, Department of Medicine, Rutgers Robert Wood Johnson Medical School, New Brunswick, NJ, USA

${ }^{14}$ Division of Pulmonary, Critical Care, and Sleep Medicine, Department of Medicine, Department of Emergency Medicine, Icahn School of Medicine at Mount Sinai, New York, NY, USA

${ }^{15}$ Division of Pulmonary and Critical Care Medicine, Rutgers New Jersey Medical School, Newark, NJ, USA

STOP-COVID investigators: A Bilal Malik, Abbas Bitar, Abeer Abu-Saif, Adam Green, Afshin Ahoubim, Ajiboye Oyintayo, Aju Jose, Akshay Athreya, Alanna L Jacobs, Alessia Fornoni, Alexander J Hodakowski, Alexander S Leidner, Alexandre M Shehata, Alfredo lardino, Allon N Friedman, Amanda D Renaghan, Amanda K Leonberg-Yoo, Amar D Bansal, Ambarish M Athavale, Amber S Podoll, Ambreen Azhar, Amee Patrawalla, Ameeka Pannu, Amy M Zhou, Anand Srivastava, Andrew J Admon, Andrew Vissing, Anip Bansal, Anitha Vijayan, Anna E Hasty, Anne K Sutherland, Anthony J Faugno, Aparna Saha, Aquino Williams, Arash Rashidi, Ariel L Mueller, Aromma Kapoor, Ashita Tolwani, Ashley Macina, Asma Tariq, Ayesha Ahmed, Barbara A Danek, Benjamin Wu, Bhavarth Shukla, Bijal Mehta, Brent R Brown, Brian T Garibaldi, Carl E Schulze, Carl P Walther, Carlos Martinez, Caroline M Hsu, Celia P Corona-Villalobos, Charles R Vasquez, Chelsea Meloche, Chintan V Shah, Chirag R Parikh, Chris Rowan, Christa A Schorr, Christina Mariyam Joy, Christopher L Mosher, Christopher Meshberger, Csaba P Kovesdy, Daniel E Weiner, Daniel L Edmonston, Danny Perry, Danyell Hall, David Charytan, David De La Zerda, David E Leaf, David Zonies, Deena R Altman, Deepa G Lazarous, Denzel Zhu, Dheeraj Reddy Sirganagari, Di Pan, Diana G Finkel, Duwayne L Willett, Eboni G Price-Haywood, Edward J Schenck, Carmen Elena Cervantes, Elizabeth Anderson, Elizabeth H Au, Ellen L Burnham, Emily A Leven, Emily H Chang, Emily J Gallagher, Eric Judd, Erik T Newman, Ernie Yap, Esha M Kapania, Ethan C Kim, Etienne Macedo, Farah Madhani-Lovely, Frank Modersitzki, Gabriel Contreras, Gabriel Naimy, Gabriela Bambrick-Santoyo, Girish N Nadkarni, Gohar Mosoyan, Goni Katz-Greenberg, Greg L Schumaker, Gregory P Milligan, H Bryant Nguyen, Hanna Berlin, Harin Rhee, Harkarandeep Singh, Hayley B Gershengorn, Heather Yang, Howard Soh, Huei Hsun Wen, Huiwen Chen, Husam Shadid, Ian A Strohbehn, Ibrahim Mohamed, Ilya Berim, Isha Puri, Ishaan Vohra, Jacqueline M Kruser, Jag Sunderram, James P Lash, Jamuna Krishnan, Jared Radbel, Jason Y Adams, Jatan A Shah, Javier A Neyra, Jay L Koyner, Jayanth S Vatson, Jean-Sebastien Rachoin, Jeffery O Boateng, Jeffrey M Paer, Jennifer E Flythe, Jennifer Griffiths, Jesus Rodriguez, Jiahua Li, Jie Ouyang, Jill H Sharma, Jing G Wang, Jingjing Zhang, Jochen Reiser, John Guirguis, John J Byun, John P Donnelly, John Wagner, Joseph E Levitt, Joy-Marie Hermes, Juan Carlos Q Velez, Juan D Valencia, Justin Arunthamakun, Jyotsana Thakkar, Jyotsna Bhattacharya, Kaltrina Sedaliu, Kalyan Prudhvi, Kapil K Pokharel, Katharine Senter, Katherine J Kelly, Kathleen D Liu, Kathleen F Kopecky, Keith M Rose, Kianoush Kashani, Kishan I Padalia, Kristen M Hess, Kusum S Mathews, Kyle B Enfield, Ladan Golestaneh, Laura Bickley, Laura Latta, Leah Meyer, Leon Boudourakis, Leslie F Thomas, Lili Chan, Lisa Shea, Luis A Matute-Trochez, Luis G GomezEscobar, Madona Elias, Maria Plataki, Mariah Thaxton, Marie Anne Sosa, Mark Liotta, Marta Christov, Mary C Mallappallil, Matthew J Tugman, Matthew T Scharf, Matthew W Semler, Maureen Brogan, Meaghan S Roche, Megan L Krajewski, Meghan E Sise, Meghan Lee, Michael Chang, Michael Pan, Michal L Melamed, Michel Chonchol, Miguel A Hernán, Miklos Z Molnar, Milagros Yunes, Min I Joo, Moh'd A Sharshir, Mohamed Farag, Molly Fisher, Mridula V Nadamuni, Mrigank S Gupta, Muhammad Imran Ali, Muner MB Mohamed, Natalie C Ernecoff, Neelja Kumar, Nicholas S Cairl, Nilam P Patel, Nitender Goyal, Noor ul aain Bhatti, Nourhan Chaaban, Omer Kamal, Pablo Garcia, Patricia F Kao, Patricia Walters, Patrick O' Hayer, Pattharawin Pattharanitima, Paul A Bergl, Pavan K Bhatraju, Pennelope Blakely, Perry Wilson, Peter A McCullough, Peter Cangialosi, Peter Hart, Pramod K Guru, Princy N Kumar, Rafey Feroze, Rajat Kapoor, Rana Hejal, Ravi K Kshirsagar, Rayan Kaakati, Rebecca Lisk, Rebecca M
Beutler, Rebecca V Levy, Richard G Wunderink, Ritesh Raichoudhury, Roberta E Redfern, Ronaldo C Go, Rubab F Malik, Rui Song, Rupali S Avasare, Ryan Mocerino, Ryan C Spiardi, S Susan Hedayati, Sabrina Taldone, Sabu John, Salim S Hayek, Samah Abu Omar, Samantha K Brenner, Samaya I Anumudu, Samir C Gautam, Samuel AP Short, Sang Hoon Woo, Sanjana Kapoor, Sanjeev Gupta, Seble G Kassaye, Sergio L Alvarez-Mulett, Seth Goldberg, Shahrzad Tehranian, Shahzad Shaefi, Shani Shastri, Shreyak Sharma, Shristi Upadhyay, Shruti Gupta, Shuchi Anand, Siddhartha Bajracharya, Siddharth Verma, Sidharth Shankar, Simon Correa, Sobaata Chaudhry, Sonali Bose, Sonika Puri, Soo Jung Cho, Sowminya Arikapudi, Stefi Lee, Stephanie M TothManikowski, Steven G Coca, Steven Menez, Steven Y Chang, Sunita Sharma, Sushrut S Waikar, Suzanne M Boyle, Tanima Arora, Tanira Ferreira, Tanveer Shaukat, Tanya S Johns, Tariq U Azam, Thuy-Duyen Nguyen, Timothy E Albertson, Tingting Li, Todd A Miano, Ugochukwu Ugwuowo, Vadym V Rusnak, Valerie Allusson, Vasantha K Jotwani, Vasil Peev, Vishal Jaikaransingh, Vivian S Cruz, Wei Wang, William Whalen, Xiaoying Deng, Yahya Ahmad, Yan Zhou, Yohannes Melaku, Yorg Azzi, Yumeng Wen, Zaza Cohen, Zoe A Kibbelaar, Zoe Post.

Contributors: SSH, DEL, and BKN designed the study. SSH did the statistical analysis. SSH, SKB, TUA, HRS, EA, HB, MP, CM, RF, PO, RK, $A B, K P, D P, P B, S G, S S, A M S, D M C, A B, M M, A S, M L M, A M S$, JS, AKS and the STOP-COVID contributors collected the data. SSH, DEL, and BKN, drafted the manuscript. SKB, TUA, HRS, EA, HB, MP, CM, RF, PO, RK, $A B, K P, D P, P B, S G, S S, A M S, D M C, A B, M M, A S, M L M, A M S, I S, K S M$, and AKS revised the manuscript for critical content. The corresponding author attests that all listed authors meet authorship criteria and that no others meeting the criteria have been omitted. SSH and DEL are the guarantors. BKN and DEL contributed equally to the manuscript. Funding: The authors of the writing committee are supported by several grants from the National Institutes of Health (NIH): 1R01HL153384 (SSH), K23HL130648 (KSM), and R01HL144566 (DEL). SSH is also supported by the Frankel Cardiovascular Center COVID-19 Impact Research Ignitor U-M G024534. The funding sponsors did not contribute to the design and conduct of the study; collection, management, analysis, or interpretation of the data; or preparation, review, approval, or decision to submit the manuscript. The content is solely the responsibility of the authors and does not necessarily represent the official views of the $\mathrm{NIH}$.

Competing interests: All authors have completed the ICMJE uniform disclosure form at www.icmje.org/coi_disclosure.pdf and declare: support from the National Institutes of Health and the Franke Cardiovascular Center COVID-19 Impact Research Ignitor for the submitted work; no financial relationships with any organizations that might have an interest in the submitted work in the previous three years; no other relationships or activities that could appear to have influenced the submitted work.

Ethical approval: The study was approved with a waiver of informed consent by the institutional review board at each participating site. Data sharing: Data from STOP-COVID are not available for sharing. The lead author (the manuscript's guarantor) affirms that the manuscript is an honest, accurate, and transparent account of the study being reported; that no important aspects of the study have been omitted; and that any discrepancies from the study as planned have been explained.

Dissemination to participants and related patient and public communities: The results of the research will be disseminated to the public through press releases, social media postings, and media commentary. We are unable to directly provide the results of the research to study participants as this study analyzed deidentified data.

Provenance and peer review: Not commissioned; externally peer reviewed.

This is an Open Access article distributed in accordance with the Creative Commons Attribution Non Commercial (CC BY-NC 4.0) license, which permits others to distribute, remix, adapt, build upon this work non-commercially, and license their derivative works on different terms, provided the original work is properly cited and the use is noncommercial. See: http://creativecommons.org/licenses/by-nc/4.0/.

1 Kramer DB, Lo B, Dickert NW. CPR in the covid-19 era - an ethical framework. N Engl J Med 2020;383:e6. doi:10.1056/ NEJMp2010758

2 Shao F, Xu S, Ma X, Xu Z, Lyu J, Ng M, et al. In-hospital cardiac arrest outcomes among patients with COVID-19 pneumonia in Wuhan, China. Resuscitation 2020;151:18-23. doi:10.1016/j. resuscitation.2020.04.005 
3 Sheth V, Chishti I, Rothman A, et al. Outcomes of inhospital cardiac arrest in patients with COVID-19 in New York City. Resuscitation 2020;155:3-5. doi:10.1016/j. resuscitation.2020.07.011

4 Ranney ML, Griffeth V, Jha AK. Critical supply shortages - the need for ventilators and personal protective equipment during the covid-19 pandemic. N Engl J Med 2020;382:e41. doi:10.1056/ NEJMp2006141

5 Hospitals consider universal do-not-resuscitate orders for coronavirus patients Washington Post2020. https://www.washingtonpost.com/ health/2020/03/25/coronavirus-patients-do-not-resucitate/.

6 Gupta S, Hayek SS, Wang W, et al. Factors associated with death in critically ill patients with coronavirus disease 2019 in the US. JAMA Intern Med 2020. doi:10.1001/jamainternmed.2020.3596

7 Harris PA, Taylor R, Minor BL, et al, The REDCap consortium. Building an international community of software platform partners. J Biomed Inform 2019;95:103208. doi:10.1016/j.jbi.2019.103208

8 Harris PA, Taylor R, Thielke R, Payne J, Gonzalez N, Conde JG. Research electronic data capture (REDCap)-a metadata-driven methodology and workflow process for providing translational research informatics support. J Biomed Inform 2009;42:377-81. doi:10.1016/j. jbi.2008.08.010

9 Vincent JL, Moreno R, Takala J, et al. The SOFA (Sepsis-related Organ Failure Assessment) score to describe organ dysfunction/failure. On behalf of the Working Group on Sepsis-Related Problems of the European Society of Intensive Care Medicine. Intensive Care Med 1996;22:707-10. doi:10.1007/BF01709751

10 Raith EP, Udy AA, Bailey M, et al. Prognostic accuracy of the SOFA score, SIRS criteria, and qSOFA score for in-hospital mortality among adults with suspected infection admitted to the intensive care unit. JAMA 2017;317:290-300. doi:10.1001/jama.2016.20328

11 Jentzer JC, Bennett C, Wiley BM, et al. Predictive value of the sequential organ failure assessment score for mortality in a contemporary cardiac intensive care unit population. J Am Heart Assoc 2018;7: e008169. doi:10.1161/JAHA.117.008169

12 Aakre C, Franco PM, Ferreyra M, Kitson J, Li M, Herasevich V. Prospective validation of a near real-time EHR-integrated automated SOFA score calculator. Int J Med Inform 2017;103:1-6. doi:10.1016/j.ijmedinf.2017.04.001

13 Stiell IG, Nesbitt LP, Nichol G, et al. Comparison of the Cerebral Performance Category score and the Health Utilities Index for survivors of cardiac arrest. Ann Emerg Med 2009;53:241-8. doi:10.1016/j.annemergmed.2008.03.018

14 Jennett B, Bond M. Assessment of outcome after severe brain damage. Lancet 1975;1:480-4. doi:10.1016/S01406736(75)92830-5

15 Ackermann M, Verleden SE, Kuehnel M, et al. Pulmonary vascular endothelialitis, thrombosis, and angiogenesis in covid-19. N Engl J Med 2020:383:120-8 doi:10.1056/NEJMoa2015432

16 Helms J, Tacquard C, Severac F, et al. High risk of thrombosis in patients with severe SARS-CoV-2 infection: a multicenter prospective cohort study. Intensive Care Med 2020;46:1089-98. doi:10.1007/ s00134-020-06062-x

17 Al-Samkari H, Karp Leaf RS, Dzik WH, et al. COVID-19 and coagulation: bleeding and thrombotic manifestations of SARS-CoV-2 infection. Blood 2020:136:489-500 doi:10.1182/blood 2020006520

18 Girotra S, Tang Y, Chan PS, Nallamothu BK. American Heart Association Get With The Guidelines-Resuscitation I. Survival after in-hospital cardiac arrest in critically ill patients: implications for covid-19 outbreak? Circ Cardiovasc Qual Outcomes 2020;13:e006837. doi:10.1161/ CIRCOUTCOMES.120.006837

Web appendix: Supplementary appendix Web appendix: Supplementary material 